\title{
Genetic Diversity of Seashore Paspalum Revealed with Simple Sequence Repeat Markers
}

\author{
Qing Shen, Hua Bian, and Hai-yan Wei \\ Institute of Tropical Crop, Hainan University, Haikou 570228, China; and Tropical Crop Germplasm \\ Research Institute, Chinese Academy of Tropical Agricultural Sciences, Haikou 571101, China \\ Li Liao \\ Institute of Tropical Crop, Hainan University, Haikou 570228, China \\ Zhi-yong Wang \\ College of Forestry, Hainan University, Haikou 570228, China
}

Xiao-yan Luo and Xi-peng Ding

Tropical Crop Germplasm Research Institute, Chinese Academy of Tropical Agricultural Sciences, Haikou 571101, China

\author{
Zhenbang Chen and Paul Raymer \\ Department of Crop and Soil Science, University of Georgia, Griffin, GA 30223
}

\begin{abstract}
Additional INDEX wORDs. germplasm, molecular marker, Paspalum vaginatum, polymorphic analysis, turfgrass
ABstract. Seashore paspalum (Paspalum vaginatum) is an important warm-season turfgrass distributed in tropical and coastal areas. It has excellent resistance to abiotic stresses, such as salinity, drought, and low temperature. However, the research on genetic diversity of local $P$. vaginatum collections from China is limited. In this study, the genetic diversity among $58 \mathrm{P}$. vaginatum accessions from four different provinces in China and four cultivars were assessed using simple sequence repeat (SSR) markers. The results indicated that a total of 45 alleles were detected by 19 polymorphic markers, with a range of 2 to 4 and an average of 2.4 alleles per marker. The genetic similarity coefficients between each pair of the $58 P$. vaginatum accessions and four cultivars ranged from 0.51 to 1.00 , with an average of 0.77 . The range of variation of Shannon diversity index of each SSR marker was 0.047 to 1.075, with an average of 0.486 . The polymorphic information content of each SSR marker varies from 0.016 to 0.577 , with an average of 0.249 . The results of cluster analysis and principal component analysis (PCA) showed that 58 P. vaginatum accessions and four cultivars were divided into four groups. These results provide the theoretical basis for the genetic diversity assessments and molecular marker-assisted breeding of $P$. vaginatum species.
\end{abstract}

Paspalum vaginatum is a warm-season perennial grass of the Gramineae family that is distributed in the coastal areas of the tropical and subtropical regions of the world (Chen et al., 2005). It mainly grows in coastal areas, mudflats, and other habitats with high salinity. It has excellent resistance to abiotic stresses, such as salinity, drought, and low temperature (Jia et al., 2015a; Shahba et al., 2014; Wu et al., 2018; Ye et al., 2010). As turfgrass, $P$. vaginatum has been widely used on golf courses, sport fields, and landscapes (Berndt, 2007). In terms of water and soil conservation, $P$. vaginatum plays a very important role for preventing soil erosion because of its ecologically aggressive and fast growing characteristics under extremely warm and humid

Received for publication 4 Nov. 2019. Accepted for publication 11 Mar. 2020. Published online 28 April 2020.

We sincerely acknowledge the editors and reviewers for their valuable comments to improve this manuscript. This work was supported by Innovative Scientific Research Project for Postgraduate students in Hainan Province in 2017 (Hys201781), Innovative Scientific Research Project for Postgraduate students in Crop Science of Hainan University in 2018 (ZWCX2018032), the Key Project of Education Department of Hainan Province (No. Hnky2019ZD-3), and Integrated Demonstration of Key Techniques for the Industrial Development of Featured Crops in Rocky Desertification Areas of Yunnan-Guangxi-Guizhou Provinces. Z.-y.W. and X.-p.D. are the corresponding authors. E-mail: wangzhiyong@hainu. edu.cn or xipding@163.com.

This is an open access article distributed under the CC BY-NC-ND license (https://creativecommons.org/licenses/by-nc-nd/4.0/). climates (Xie et al., 2004). It also has been used for the bioremediation of contaminated soil and to improve the productivity of soil [e.g., heavy metals and organic chemicals (Duan et al., 2016; Wang et al., 2010)].

As an important warm-season turfgrass, there have been many studies on $P$. vaginatum, such as salt tolerance, drought resistance, and cadmium tolerance (Chen et al., 2012; Wu et al., 2015; Xie and Lu, 2004; Xu et al., 2018). However, there were few reports on genetic diversity of $P$. vaginatum in recent years. Liu et al. (1994) used random amplified polymorphic DNA (RAPD) to detect the genetic diversity of $46 P$. vaginatum ecotypes. The genetic diversity, size, and distribution pattern of three wild accessions and one cultivar of $P$. vaginatum from Guangdong Province were analyzed by RAPD molecular marker technique (Xie et al., 2004). Four introduced cultivars and one thick-stem mutant of $P$. vaginatum were assessed using morphological and amplified fragment length polymorphism (AFLP) analysis; the results showed that the genetic distance of morphology was consistent with the clustering result of similarity coefficient of AFLP markers (He et al., 2011). The optimization of sequence-related amplified polymorphism (SRAP) molecular marker reaction system and the selection of primers of $P$. vaginatum were carried out based on four typical $P$. vaginatum germplasms (Liu et al., 2016). Molecular markers have been proven to be powerful tools to assess the 
genetic diversity of $P$. vaginatum for germplasm collection and plant breeding programs. These studies can promote the development of genetic diversity of $P$. vaginatum.

Compared with other molecular markers, SSR markers have been widely used in many fields of plant research due to their high frequency, high polymorphism, repeatability, codominant inheritance, and simple methodology (Sun et al., 2011). Recently, SSR markers have been applied to detect the genetic diversity in turfgrasses such as Cynodon dactylon (Long et al., 2016) and Zoysia japonica (Guo et al., 2008); however, limited SSR sequence information is available for $P$. vaginatum, which restricts the development of molecular markers and analysis of genetic diversity. Therefore, more SSR markers need to be developed for characterization and evaluation of $P$. vaginatum. Wang et al. (2006) selected and used 40 SSR markers from wheat (Triticum aestivum), maize (Zea mays), and sorghum (Sorghum bicolor) to genetically distinguish $73 P$. vaginatum accessions. Harris-Shultz et al. (2013) used 80 SSR markers to assess genetic relationships among $18 P$. vaginatum accessions. Li et al. (2016) developed 50 pairs of expressed sequence tag (EST)-SSR primers and selected 10 turfgrass cultivars to test the validity and polymorphism of these primers.

In addition to the introduction and cultivation of $P$. vaginatum in China, the research, development, and utilization of local resources are extremely rare. To accelerate the efficient development and utilization of $P$. vaginatum, SSR molecular marker technology was used to analyze the genetic diversity of local $P$. vaginatum germplasm in China in this study. These results provide the theoretical basis for the genetic diversity and molecular marker-assisted breeding of $P$. vaginatum species.

\section{Materials and Methods}

Plant materials. The experimental materials were 62 $P$. vaginatum germplasm resources, including $58 P$. vaginatum accessions collected from four different provinces in China, of which 36 accessions were collected from Hainan province, 14 accessions were collected from Guangxi province, seven were collected from Guangdong province, and one was collected from Jiangxi province, plus an additional four cultivars from the University of Georgia (Table 1).

All materials were propagated from healthy stolons gathered from their native habitat and planted in the experimental field of Danzhou campus of Hainan University. Materials were collected according to geographical distribution, the distance between the materials was $2 \mathrm{~km}$. Materials from the same or adjacent regions are collected according to morphological differences. The experimental materials were planted according to the serial number sequence, the stolon segment of each accession was planted in separate $1 \times 1-\mathrm{m}$ plots. The distance between the individual plots was $50 \mathrm{~cm}$. The plot edges were trimmed twice weekly to prevent cross contamination between adjacent plots. Routine irrigation and fertilization were carried out to maintain healthy growth.

EXTRACTION, AMPLIFICATION, AND ELECTROPHORETIC DETECTION OF DNA. The DNA was extracted from fresh leaves of $P$. vaginatum by the cetyl trimethylammonium bromide method (Doyle and Doyle, 1987). The quality and concentration of DNA were measured by $1.0 \%$ agarose gel electrophoresis. Samples were diluted with sterile water to a final concentration of $100 \mathrm{ng} \cdot \mu \mathrm{L}^{-1}$ and stored at $-20{ }^{\circ} \mathrm{C}$.

SSR AMPLIFICATION. In this study, 58 SSR markers previously reported by Jia et al. (2015b) and Li et al. (2016) were evaluated in $P$. vaginatum. Four $P$. vaginatum accessions (USA17-4, USA17-29, HN17-22, and HN7-54) were randomly selected to screen the 58 SSR markers. The markers with clear and distinct bands were used for polymerase chain reaction (PCR) amplification of $58 \mathrm{P}$. vaginatum accessions and four cultivars (Table 2). The PCR amplification system and procedures were performed according to the method by Jiang (2017). For PCR amplification, a $20-\mu \mathrm{L}$ reaction with $1.0 \mu \mathrm{L}$ DNA $\left(100 \mathrm{ng} \cdot \mu \mathrm{L}^{-1}\right), 1.0 \mu \mathrm{L}$ primers $\left(5 \mu \mathrm{mol} \cdot \mathrm{L}^{-1}\right), 0.3 \mu \mathrm{L}$ Taq DNA polymerase $\left(5 \mathrm{U} \cdot \mu \mathrm{L}^{-1}\right), 1.4 \mu \mathrm{L}$ deoxynucleoside triphosphates $\left(2.5 \mathrm{mmol} \cdot \mathrm{L}^{-1}\right), 2.0 \mu \mathrm{L} 10 \times$ PCR buffer, and $14.3 \mu \mathrm{L}$ doubledistilled $\mathrm{H}_{2} \mathrm{O}$ was used. The amplification program was as follows: 4 min at $94{ }^{\circ} \mathrm{C}$; followed by 38 cycles of $94^{\circ} \mathrm{C}$ for $40 \mathrm{~s}$, $55^{\circ} \mathrm{C}$ for $45 \mathrm{~s}$, and $72{ }^{\circ} \mathrm{C}$ for $45 \mathrm{~s}$; with a final extension at $72{ }^{\circ} \mathrm{C}$ for $5 \mathrm{~min}$; and then the reaction was cooled to $4{ }^{\circ} \mathrm{C}$. The amplification products were separated on an $8 \%$ nondenaturing polyacrylamide gel, which was electrophoresed at a voltage of $140 \mathrm{~V}$ for $3 \mathrm{~h}$. Then the gel was silver stained (Li et al., 2004), developed with formaldehyde solution, and finally photographed and recorded.

DAta PROCESSing AND ANALYsis. The SSRs generated by PCR were manually scored using a 50-base pair DNA ladder marker as a reference. Spreadsheet software (Excel; Microsoft, Redmond, WA) was used to conduct the " 0 , 1" system statistics on each band, with band presence marked as " 1 " and absence marked as " 0 ", to generate the original data matrix. Following the method proposed by Nei and Li (1979), the distance matrix and system tree diagram of the tested material were calculated using NTSYS-pc software [version 2.10e (Rohlf, 2000)]. Genetic similarity cluster analysis was conducted according to unweighted pair group method using arithmetic average [UPGMA (Sneath and Sokal, 1973)]. POPGENE 1.32 software (Yeh et al., 1999) was used to calculate the genetic diversity parameters of each marker, package the number of alleles (Na), Shannon polymorphism index (I), observed heterozygosity (Ho), and excepted heterozygosity (He) (Nei, 1973). Polymorphic information content (PIC) was calculated by using the frequency of each allele of SSR marker according to the method of Botstein et al. (1980). The formula is as follows:

$$
P I C=1-\sum_{i=l}^{n} P_{i}^{2}-\sum_{i=l}^{n-l} \sum_{j=i+l}^{n} 2 P_{i}^{2} P_{j}^{2}
$$

In the formula, $n$ represents the number of alleles detected by each marker, and $P_{i}, P_{j}$ represent the frequency of the presence of the $i, j$ alleles in the tested materials.

\section{Results}

GenetiC Diversity. In this study, 27 markers with clear and distinct bands were selected from the 58 SSR markers. Using PCR to amplify the 27 markers, we found that 19 SSR markers were polymorphic (Table 2 ) in our 62 P. vaginatum samples. A total of 45 alleles were detected, with a range of 2 to 4 and an average of 2.4 alleles per marker (Fig. 1, Table 3). The markers with the most alleles were SP-18 and SP-37. The range of Ho of 19 SSR markers was 0.000 to 0.694 , with an average of 0.241 . The He ranged from 0.016 to 0.657 , with an average of 0.295 (Table 3). The Ho and the He of primer ESSR3 were the highest. The range of variation of I of each SSR marker was 0.047 to 1.075 , with an average of 0.486 . The PIC of each SSR 


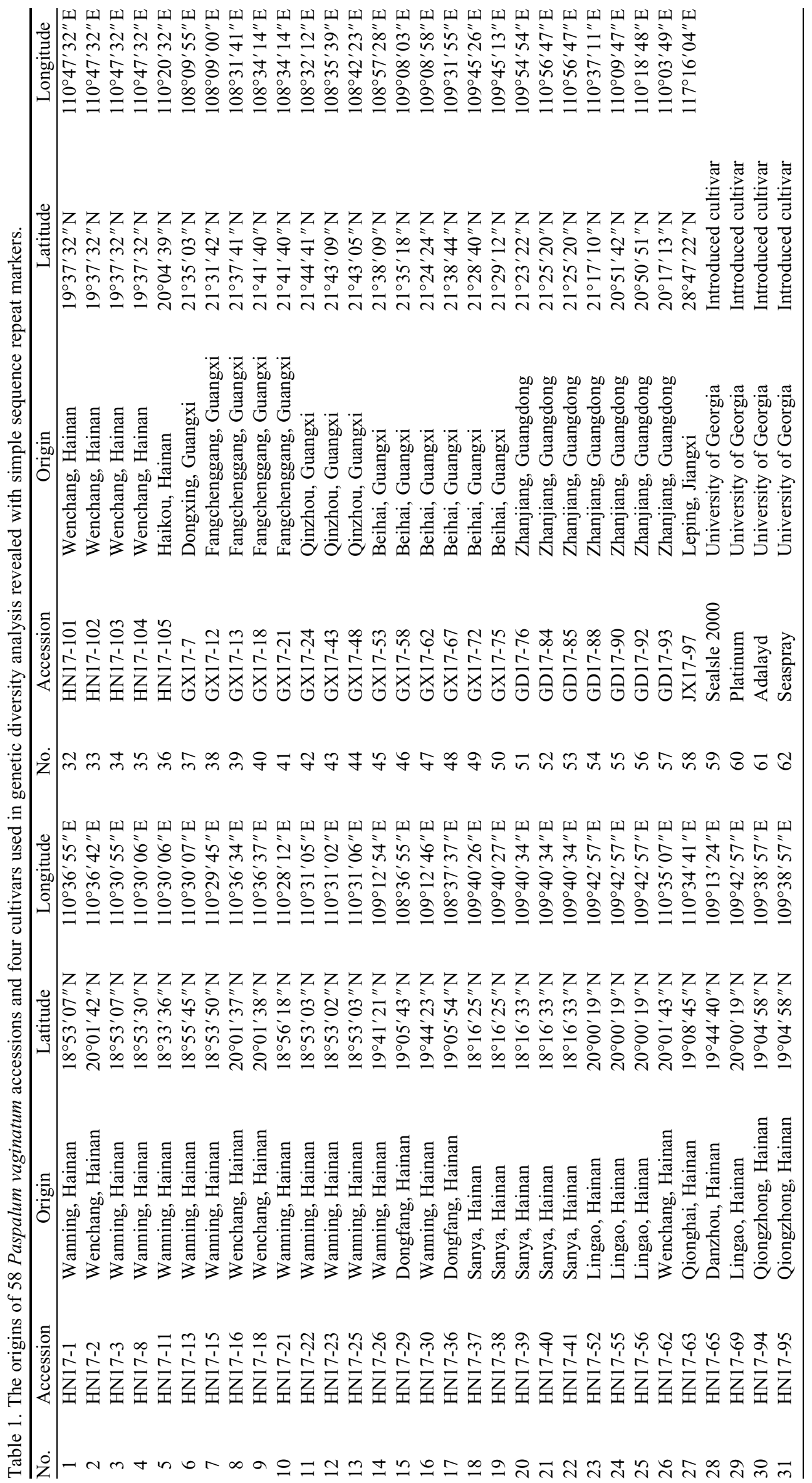




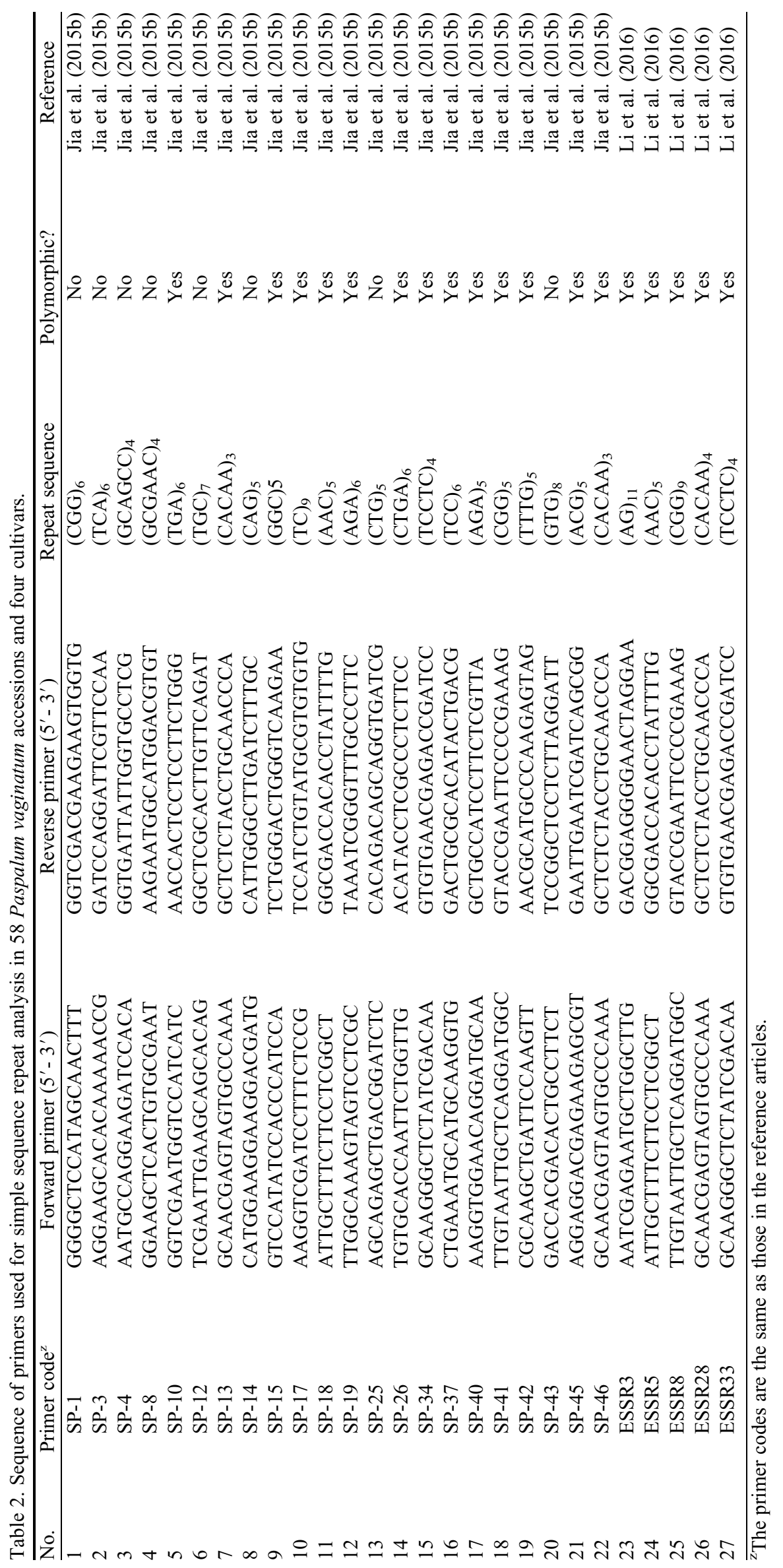




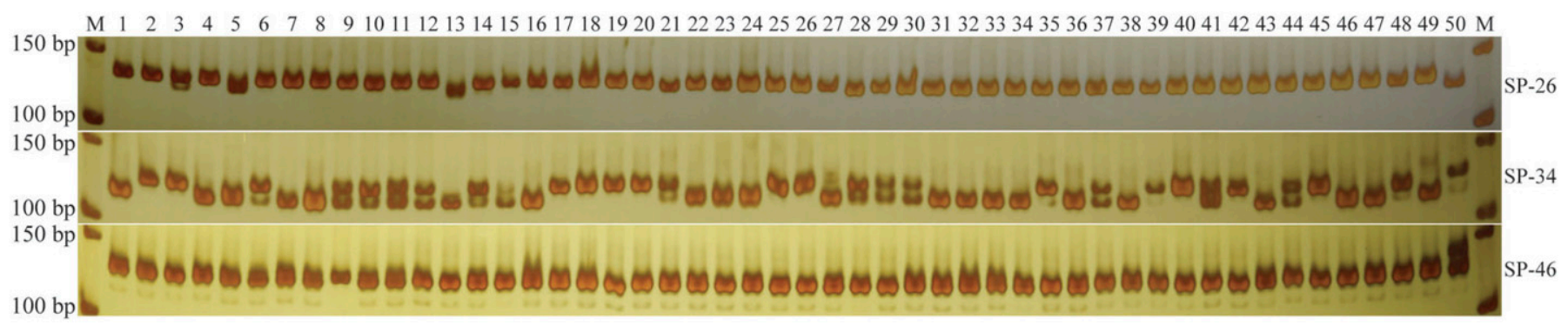

Fig. 1. Amplification profiles of part of simple sequence repeat (SSR) primers in the 50 Paspalum vaginatum accessions [lane $\mathrm{M}=50-$ base pair (bp) DNA ladder marker]. SSR profiles of 50 P. vaginatum accessions with primers SP-26, SP-34, and SP-46 (lanes 1-50).

marker varies from 0.016 to 0.577 , with an average of 0.249 (Table 3). The I and the PIC were the highest in primer ESSR3 and the lowest in primer SP-15, which indicated that ESSR3 had higher detection efficiency of polymorphism.

Cluster analysis. Based on the data of 19 polymorphic SSR markers, the genetic similarity coefficients (GSCs) among different materials were calculated. The GSCs between each pair of the $58 P$. vaginatum accessions and four cultivars ranged from 0.51 to 1.00 , with an average of 0.77 . The lowest GSC (0.51) existed between HN17-2 and HN17-26 populations, indicating that they were least related. Cluster analysis based on GSCs showed that the 58 P. vaginatum accessions and four cultivars were classified into four distinct major groups: A, B, C, and D (Fig. 2) using a similarity index of 0.75 . Most of the accessions or cultivars collected from the same region or nearby regions had high genetic similarity and tended to cluster within the same group or neighboring groups; however, there was no significant relationship between genetic diversity and geographic location. Cluster A contained six $P$. vaginatum accessions: one from Guangxi (GX17-62) and five from Hainan province (Fig. 2). Cluster B included 29 P. vaginatum accessions and four cultivars, which was divided into two subgroups [B1 and B2 (Fig. 2)]. The subgroup B1 contained 25 accessions, including 14 Hainan accessions, 7 Guangxi accessions, 3 Guangdong accessions, 1 Jiangxi accession (JX17-97), and two cultivars (Seaspray and Adalayd) (Fig. 2). Subgroup B2 included four Hainan accessions and two cultivars (Platinum and Sealsle 2000). Cluster C consisted of 21 P. vaginatum accessions, and could be classified further into three relatively distinct subgroups of $\mathrm{C} 1, \mathrm{C} 2$, and $\mathrm{C} 3$ (Fig. 2). The subgroup C1 included 1 Hainan accession and 2 Guangxi accessions; the subgroup C2 comprised 15 accessions, which were 11 Hainan accessions, 2 Guangxi, and 2 Guangdong accessions; and the subgroup C3 contained 1 Hainan accession and 2 Guangdong accessions (Fig. 2). Cluster D included GX17-13 and GX17-48, both from Guangxi. According to the clustering results, the materials from the same area were not completely clustered in one group, so there were large genetic differences among the tested materials.

Principal component analysis. Based on the genetic resemblance matrix, the PCA was conducted on 58 P. vaginatum accessions and four cultivars to further understand the ecological distribution of different accessions. Figure 3 presents the distribution of the different accessions according to the three principal axes of variation using PCA. The percentages of variance revealed by principal component 1 (PC1), principal

Table 3. Polymorphic analysis of simple sequence repeat markers within 58 Paspalum vaginatum accessions and four cultivars.

\begin{tabular}{|c|c|c|c|c|c|c|}
\hline No. & Primer code & Alleles (no.) & Observed heterozygosity & Expected heterozygosity & Shannon index & Polymorphic information content \\
\hline$\overline{1}$ & SP-10 & 2 & 0.161 & 0.150 & 0.280 & 0.137 \\
\hline 3 & SP-15 & 2 & 0.016 & 0.016 & 0.047 & 0.016 \\
\hline 5 & SP-18 & 4 & 0.177 & 0.564 & 0.941 & 0.468 \\
\hline 6 & SP-19 & 2 & 0.177 & 0.163 & 0.300 & 0.149 \\
\hline 7 & SP-26 & 2 & 0.000 & 0.462 & 0.650 & 0.353 \\
\hline 9 & SP-37 & 4 & 0.436 & 0.416 & 0.808 & 0.384 \\
\hline 10 & SP-40 & 2 & 0.307 & 0.262 & 0.428 & 0.226 \\
\hline 11 & SP-41 & 2 & 0.048 & 0.048 & 0.114 & 0.046 \\
\hline 12 & SP-42 & 2 & 0.032 & 0.032 & 0.083 & 0.031 \\
\hline 13 & SP-45 & 3 & 0.355 & 0.370 & 0.652 & 0.326 \\
\hline 14 & SP-46 & 2 & 0.355 & 0.294 & 0.468 & 0.249 \\
\hline \multirow[t]{2}{*}{19} & ESSR33 & 2 & 0.371 & 0.446 & 0.635 & 0.345 \\
\hline & Mean & 2.4 & 0.241 & 0.295 & 0.486 & 0.249 \\
\hline
\end{tabular}




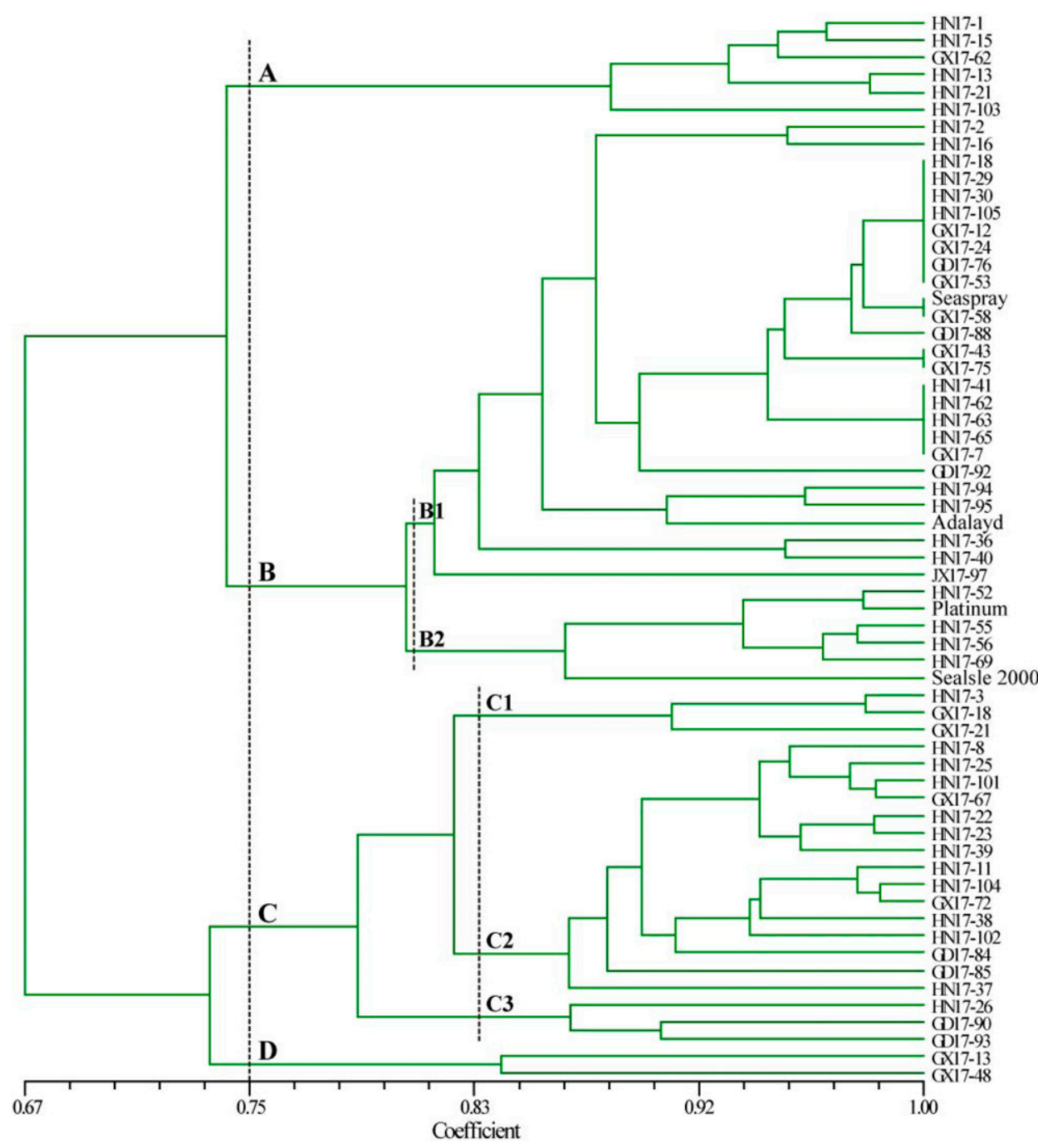

Fig. 2. Unweighted pair group method using arithmetic average clustering for 58 Paspalum vaginatum accessions and four cultivars revealed with simple sequence repeat (SSR) markers. The 58 P. vaginatum accessions and four cultivars were divided into four groups (A, B, C, and D) with the cluster analysis. Group B was divided into two subgroups of B1 and B2. Group C was classified into three subgroups of C1, C2, and C3.

diversity studies of $P$. vaginatum. At present, 220 SSR markers have been developed and applied in $P$. vaginatum (Harris-Shultz et al., 2013; Jia et al., 2015b; Li et al., 2016; Liu et al., 1995; Wang et al., 2006). In this study, a total of 45 alleles were detected by 19 polymorphic SSR markers, with a range of 2 to 4 and an average of 2.4 alleles per marker. There were fewer alleles detected in our experiment compared with the studies of Wang et al. (2006), in which they found 209 alleles using 40 SSR markers, with an average of five alleles per marker. The higher the PIC value, the more effective the primer is in evaluating genetic diversity (Smith et al., 2000). The PIC was the highest in primer ESSR3, indicating that ESSR3 has high detection efficiency of genetic diversity. The PIC for 58 P. vaginatum accessions and four cultivars was lower than that reported by Harris-Shultz et al. (2013), which ranged from 0.00 to 0.78 and with an average value of 0.31 for all markers. The differences observed might be due to the different SSR markers used in the two studies, or the fact that the accessions used in their studies were collected from much more diverse areas, even different continents (Budak et al., 2004). Therefore, SSR technology is an effective method for genetic diversity analysis of $P$. vaginatum.

Cluster analysis and PCA, based on these SSR markers, separated all

component 2 (PC2), and principal component 3 (PC3) were $76.50 \%, 9.24 \%$, and $3.30 \%$, respectively. According to the first three principal components of 62 materials, the PCA threedimensional scattered point distribution map (Fig. 3) was consistent with the results of the UPGMA cluster analysis. Fifty-eight $P$. vaginatum accessions and four cultivars were also divided into four groups, of which group A was more concentrated, whereas groups $\mathrm{B}$ and $\mathrm{C}$ were relatively scattered. In contrast, PCA (Fig. 3) is more intuitive than cluster analysis (Fig. 2) in revealing the genetic relationships among different materials.

\section{Discussion and Conclusions}

Molecular markers are important tools for plant genetic research and molecular marker-assisted breeding (Ding et al., 2015). Compared with the other molecular markers based on PCR, SSR markers are abundant in quantity, highly polymorphic, codominant, and user friendly (Bian et al., 2019). SSR makers have been applied in many fields (Ding et al., 2015). SSR markers have previously been used successfully in genetic
58 P. vaginatum accessions and four cultivars into four groups in this study. These results were inconsistent with the findings of Harris-Shultz et al. (2013) and Wang et al. (2006), which were clustered into two and three major groups, respectively. The different results may be due to the different sources of experimental materials. The experimental materials of this study were mainly concentrated in Hainan, Guangdong, and Guangxi provinces, which were very close to each other. Most of the accessions or cultivars, which were collected from the same region or nearby regions, had higher GSCs and tended to cluster within the same group or neighboring groups (Wang et al., 2013). The four cultivars had a high level of genetic similarity in the study. However, the accessions from the same area were not completely grouped together. For example, Guangxi accessions were distributed in all four groups, and Hainan accessions were also distributed in three groups (groups A, B, and C). Similar results were also reported by Wang et al. $(2011,2013)$. In addition, there were accessions from different regions clustered in the same group. For instance, Hainan, Guangxi, and Guangdong accessions were clustered in group B or C. This also appeared in other species (Liao et al., 2016; 


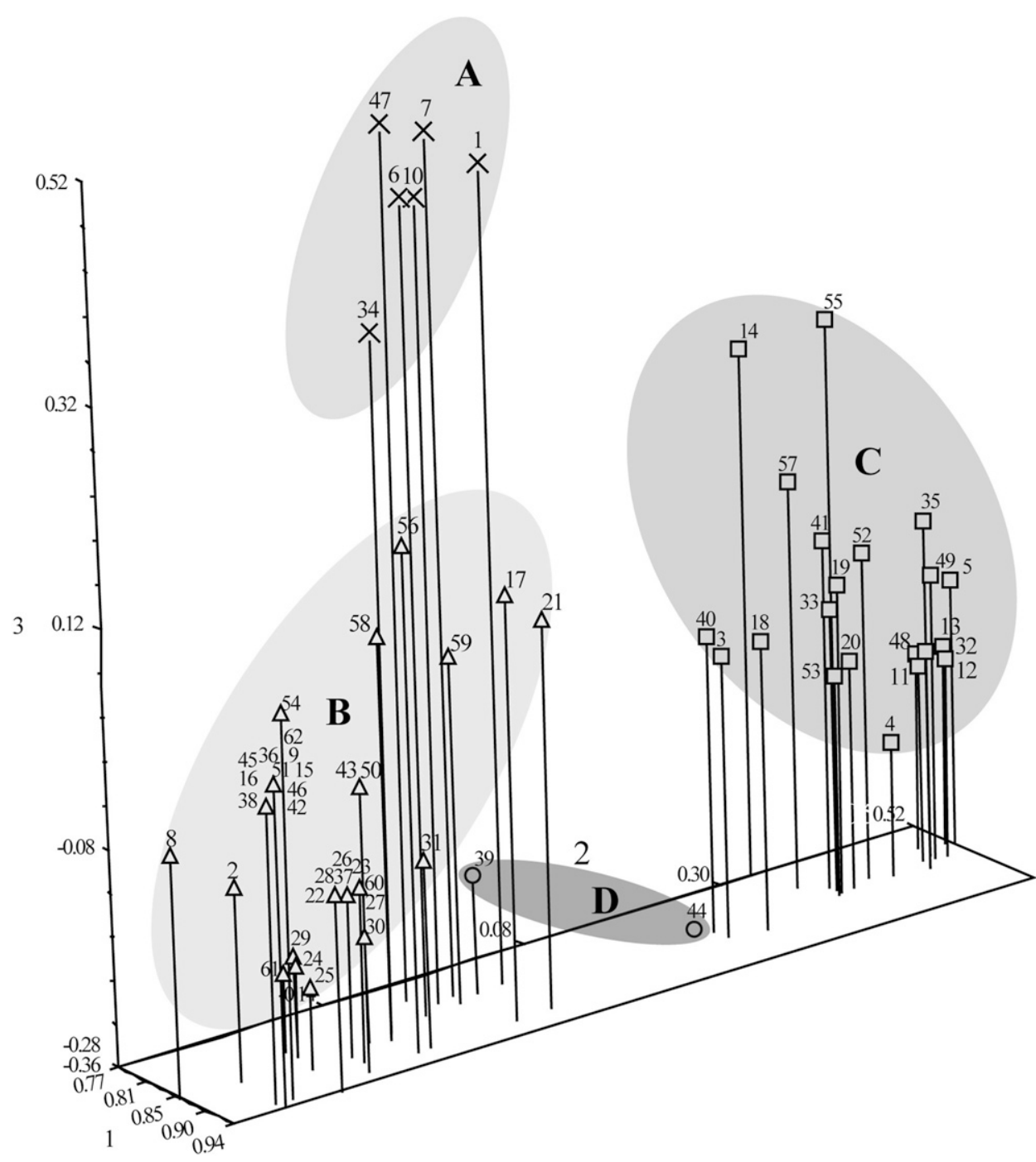

Fig. 3. Three-dimensional scatter plot with the principal component analysis (PCA) for 58 Paspalum vaginatum accessions and four cultivars revealed with simple sequence repeat markers. The numbers for materials in the figure are corresponding to the numbers of accessions given in Table 1 . The 58 P. vaginatum accessions and four cultivars were divided into four groups $(\mathrm{A}, \mathrm{B}, \mathrm{C}$, and D) with the PCA. The groups A, B, C, and D in the figure are consistent with those in Fig. 2.

Wang et al., 2015). The clustering results of most materials were not strictly consistent with the geographical sources, which were largely due to the limitations of the sampling. These results showed that most of the accessions or cultivars from similar or adjacent regions were clustered in one group but some of the accessions or cultivars from the similar or adjacent regions were not clustered together. There were many reasons to explain these phenomena. First, $P$. vaginatum accessions or cultivars were propagated by a single asexual reproduction in the world (Evers and Burson, 2004). P. vaginatum asexual reproduction was usually spread by stolons. Second, different habitats and environments were clustered together by consciously artificial or unconscious introduction, such as the material collected by the roadside might be a cultivar that has been widely used. The materials were collected according to the geographic location, so it was possible to accidentally collect similar material during sampling. Third, there was a lack of sufficient materials and primers in this study. A large number of materials will be collected to accurately evaluate the polymorphism of $P$. vaginatum by enough SSR markers in the future. In conclusion, SSR markers were successfully used to study the genetic diversity of $P$. vaginatum, which provided an important theoretical basis for the collection of turfgrass germplasm and genetic breeding.

\section{Literature Cited}

Berndt, W.L. 2007. Salinity affects quality parameters of 'SeaDwarf' seashore paspalum. HortScience 42:417420.

Bian, H., Q. Shen, H.Y. Wei, Y.J. Jiang, X.P. Ding, and C.J. Bai. 2019. Genetic diversity and population structure analysis of Stylosanthes germplasm resources based on SSR markers. Pratacultural Sci. 36:729-743.

Botstein, D., R.L. White, M. Skolnick, and R.W. Davis. 1980. Construction of a genetic linkage map in man using restriction fragment length polymorphisms. Amer. J. Hum. Genet. 32:314331.

Budak, H., R.C. Shearman, I. Parmaksiz, and I. Dweikat. 2004. Comparative analysis of seeded and vegetative biotype buffalograsses based on phylogenetic relationship using ISSRs, SSRs, RAPDs, and SRAPs. Theor. Appl. Genet. 109:280-288.

Chen, J.B., X.Q. Chu, S. Li, J.Q. Zong, D. Wang, and J.X. Liu. 2012. Effects of saline water irrigation on growth of 7 genera and 11 species of warm season turfgrasses and their salinity tolerance difference. Pratacultural Sci. 29:1185-1192.

Chen, Z., W. Kim, M. Newman, M. Wang, and P. Raymer. 2005. Molecular characterization of genetic diversity in the USDA seashore paspalum germplasm collection. Intl. Turf. Soc. 10:543-549.

Ding, X.P., X.Y. Luo, L. Zhang, W.Q. Wang, and C.J. Bai. 2015. Genetic relationships among Stylosanthes species revealed by SSR analysis. Caoye Kexue 32:1594-1602.

Doyle, J.J. and J.L. Doyle. 1987. A rapid DNA isolation procedure for small quantities of fresh leaf tissue. Phytochem. Bull. 19:11-15.

Duan, R.J., C.B. Wu, L. Wang, J.C. Guo, S.P. Fu, J. Liu, and R.M. Li. 2016. Contents of proline, soluble sugar and chlorophyll and accumulations of nitrogen, phosphorus and potassium in Paspalum vaginatum Sw. in response to Cd stress. Jiangsu J. Agr. Sci. 32:357-361.

Evers, G.W. and B.L. Burson. 2004. Dallisgrass and other paspalum species, p. 681-713. In: L.E. Moser, B.L. Burson, and L.E. Sollenberger (eds.). Warm-season $\left(\mathrm{C}_{4}\right)$ grasses. Agron. Monogr. 45. ASA, CSSA, SSSA, Madison, WI.

Guo, H.L., J.X. Liu, Z.F. Zhou, and J.P. Xuan. 2008. Interspecific relationship and genetic diversity of zoysiagrass revealed by SSR markers. Acta Agrestia Sinica 16:552-557.

Harris-Shultz, K., P.L. Raymer, B.E. Scheffler, and R.S. Arias. 2013. Development and characterization of seashore paspalum SSR markers. Crop Sci. 53:2679-2685.

He, X.X., Y.M. Liu, and Z.L. Wang. 2011. Morphological characteristics and AFLP analysis in seashore paspalum (Paspalum vaginatum) varieties. Acta Agrestia Sinica 19:164-170. 
Jia, X.P., Y.M. Deng, X.B. Sun, and L.J. Liang. 2015a. Impacts of salt stress on the growth and physiological characteristics of Paspalum vaginatum. Acta Pratacultural Sinica 24:204-212.

Jia, X.P., Y.M. Deng, X.B. Sun, L.J. Liang, and X.Q. Ye. 2015 b. Characterization of the global transcriptome using Illumina sequencing and novel microsatellite marker information in seashore paspalum. Genes Genomics 37:77-86.

Jiang, Y.J. 2017. Construction of core collection in Stylosanthes germglasm. Hainan Univ., Haikou, China.

Li, H.Y., X.S. Wang, Y.P. Wang, Y. Wu, Q. Shen, and X.P. Jia. 2016. Analysis of SSR information in EST resources and development of ESTSSR marker in Paspalum vaginatum. Chin. Agr. Sci. Bull. 32:96-102.

Li, Y.Q., H.W. Li, L.F. Gao, and B.R. He. 2004. Progress of simple sequence repeats derived from expressed sequence tags. J. Plant Genet. Resour. 5:91-95.

Liao, L., X.L. Wang, J.X. Liu, Y. Liu, C.J. Bai, Y.J. Xu, X.Y. Zhang, and Z.Y. Wang. 2016. Analysis of genetic diversity of Axonopus compressus using ISSR markers. Pratacultural Sci. 33:608-614.

Liu, Y., Y. Wang, J.J. Ma, J.A. Shi, Z.Y. Wang, J.X. Liu, and H.L. Guo. 2016. Optimization of SRAP-PCR system in Paspalum vaginatum and primers screening. Acta Agrestia Sinica 24:1080-1086.

Liu, Z.W., R.L. Jarret, R.R. Duncan, and S. Kresovich. 1994. Genetic relationships and variation among ecotypes of seashore paspalum (Paspalum vaginatum) determined by random amplified polymorphic DNA markers. Genome 37:1011-1017.

Liu, Z.W., R.L. Jarret, S. Kresovich, and R.R. Duncan. 1995. Charaterization and analysis of simple sequence repeat (SSR) loci in seashore paspalum (Paspalum vaginatum Swartz). Theor. Appl. Genet. 91:47-52.

Long, T., C.Q. Huang, and G.D. Liu. 2016. Optimization of SSR-PCR reaction system for Cynodon dactylon by orthogonal design. Genomics Appl. Biol. 35:198-205.

Nei, M. 1973. Analysis of gene diversity in subdivided populations. Proc. Natl. Acad. Sci. USA 70:3321-3323.

Nei, M. and W.H. Li. 1979. Mathematical model for studying genetic variation in terms of restriction endonucleases. Proc. Natl. Acad. Sci. USA 76:5269-5273.

Rohlf, F.J. 2000. NTSYS-pc: Numerical taxonomy and multivariate analysis system, version 2.1 Exeter Software. Setauket, NY.

Shahba, M.A., M.S. Abbas, and S.F. Alshamary. 2014. Drought resistance strategies of seashore paspalum cultivars at different mowing heights. HortScience 49:221-229.

Smith, J.S.C., S. Hopkins, M.S. Mitchell, S.E. Dean, R.E. Woodman, W.L. Lee, and K. Porter. 2000. Genetic diversity among elite sorghum inbred lines assessed with simple sequence repeats. Crop Sci. 40:226-232.

Sneath, P.H. and R.R. Sokal. 1973. Numerical taxonomy: The principles and practice of numerical classification. 1st ed. Freeman, San Francisco, CA.
Sun, Q.M., W.C. Ma, S.P. Ma, J.S. Zhao, L.J. Bai, J.Z. Chen, C.H. Cai, X. Xiang, and L.X. Ou. 2011. Characteristics of SSRs derived from ESTs and development of EST-SSR markers in litchi (Litchi chinensis Sonn.). Sci. Agr. Sinica 44:4037-4049.

Wang, K., Y.M. Liu, and Z.L. Wang. 2010. Physiological responses of seashore paspalum and centipedegrass to soil lead contamination stresses. Pratacultural Sci. 27:32-38.

Wang, M.L., Z.B. Chen, N.A. Barkley, M.L. Newman, W. Kim, P. Raymer, and G.A. Pederson. 2006. Characterization of seashore paspalum (Paspalum vaginatum Swartz) germplasm by transferred SSRs from wheat, maize and sorghum. Genet. Resources Crop Evol. 53:779-791.

Wang, X.L., L. Liao, X.Y. Zhang, C.J. Bai, and Z.Y. Wang. 2015. Genetic diversity of carpetgrass germplasm based on simple sequence repeat markers. HortScience 50:797-800.

Wang, Z.Y., L. Liao, X.J. Yuan, A.G. Guo, and J.X. Liu. 2011. Genetic relationships of bermudagrass (Cynodon dactylon var. dactylon) from different countries revealed by sequence-related amplified polymorphism (SRAP) analysis. Afr. J. Biotechnol. 10:17106-17115.

Wang, Z.Y., L. Liao, X.J. Yuan, H.L. Guo, A.G. Guo, and J.X. Liu. 2013. Genetic diversity analysis of Cynodon dactylon (bermudagrass) accessions and cultivars from different countries based on ISSR and SSR markers. Biochem. Syst. Ecol. 46:108-115.

Wu, C.B., J.C. Guo, S.P. Fu, J. Liu, R.M. Li, L. Wang, and R.J. Duan. 2015. Physiological responses of Paspalum vaginatum $\mathrm{Sw}$. to $\mathrm{Cd}$ stress and its $\mathrm{Cd}$ accumulation. Jiangsu J. Agr. Sci. 31:1337-1343.

Wu, X.L., H.F. Shi, and Z.F. Guo. 2018. Overexpression of a NF-YC gene results in enhanced drought and salt tolerance in transgenic seashore paspalum. Front. Plant Sci. 9:1355.

Xie, X.M. and X.L. Lu. 2004. Good properties and values for utilization of seashore paspalum germplasm resource. J. South China Agr. Univ. 25:64-67.

Xie, X.M., X.L. Lu, X.S. Sun, Z.X. Dong, and H.R. Guo. 2004. Genetic diversity of seashore paspalum (Paspalum vaginatum) based on RAPD molecular markers. J. South China Agr. Univ. $\mathrm{S} 2: 10-15$.

Xu, Y.H., X.C. Xie, T.Z. Liu, and J.M. Zhang. 2018. The evaluation on drought resistance of mutants of Paspalum vaginatum. Chin. J. Trop. Crop. 39:246-253.

Ye, X.Q., J.M. She, L.F. Liang, X. Zhang, S.F. Wang, M.Q. Dong, and Y.Y. Wu. 2010. Acquirement of chilling tolerant mutant by somatic screening of low temperature in sea dallisgrass (Paspalum vaginatum Sw.). Acta Agrestia Sinica 18:97-102.

Yeh, F.C., R.C. Yang, and T. Boyle. 1999. POPGENE, version 1.32: The user friendly software for population genetic analysis. Univ. Alberta, Mol. Biol. Biotechnol. Ctr., Edmonton, AB, Canada. 